\title{
The reflection of family relations in the novel of A. Chulpan "Kecha va kunduz"
}

\section{Zilola SAYITKULOVA ${ }^{1}$}

Teacher of secondary school No. 32 of Samarkand

\begin{tabular}{l} 
ARTICLE INFO \\
\hline Article history: \\
Received February 2021 \\
Received in revised form \\
20 February 2021 \\
Accepted 15 March 2021 \\
Available online \\
15 April 2021 \\
\hline
\end{tabular}

Keywords:

work of fiction, realistic literature, marital relations,

features of Uzbek women.

\section{ABSTRACT}

The present article analyzes the relationship of couple in the early twentieth century, using the heroes of the great Uzbek novel "Kecha va Kunduz", created by A.Chulpan. The relationship of couple is interpreted by three different age characters in the novel: Zebi is a young Uzbek woman, Miryokub's wife is a middle-aged Uzbek woman, and Qurbonbibi is an older Uzbek woman.

\section{1-1415/C 2021 in Science LLC.}

This is an open access article under the Attribution 4.0 International (CC BY 4.0) license (https://creativecommons.org/licenses/by/4.0/deed.ru)

\section{A. Cho'lponning "Kecha va kunduz" romanida oilaviy munosabatlarning aks ettirilishi}

\section{Kalit so'zlar:}

badiiy asar,

realistik adabiyot,

er-xotin munosabatlari,

o'zbek ayollari xususiyatlari.
ANNOTATSIYA

Maqolada A. Cho'lpon tomonidan yaratilgan buyuk o'zbek romani "Kecha va kunduz" qahramonlaridan foydalanib, $\mathrm{XX}$ asr boshidagi er-xotin munosabatlari tahlil etilgan. Er-xotin munosabatlari romanning uch xil yoshdagi qahramonlarida talqin etigan bo'lib: Zebi - yosh o'zbek ayoli, Miryoqubning xotini - o'rta yosh o'zbek ayoli va Qurbonbibi - katta yoshdagi o'zbek ayoli sifatida olinib, ulardagi o'ziga xos va umumiy jihatlar ko'rsatib berilgan.

\footnotetext{
1 teacher of secondary school No. 32 of Samarkand, Uzbekistan
} 


\section{Отражение семейных отношений в произведении А. Чулпана «Кеча ва кундуз»}

\author{
Ключевые слова: \\ художественное \\ произведение, \\ реалистическая \\ литература, \\ супружеские отношения, \\ особенности узбекских \\ женщин.
}

\begin{abstract}
АННОТАЦИЯ
В данной статье анализируются семейные отношения в начале XX века на примере героев великого узбекского романа «Кеча ва Кундуз», созданного А. Чулпаном. Семейные, супружеские отношения интерпретируются тремя разновозрастными персонажами романа: Зеби молодая узбекская девушка, жена Мирёкуба - узбекская женщина средних лет, а Курбонбиби - узбекская женщина старшего поколения.
\end{abstract}

\section{KIRISH}

Umrboqiy asarlar sirasiga mansub, Abdulhamid Sulaymon o'g'li Cho'lponning "Kecha va Kunduz" asari o'zbek romanchiligi tarixida o'z alohida o'rniga ega asardir. U o'zining tabiiyligi, samimiyligi, ta'sirchanligi mazmunining teranligi va xalq hayotini barcha ranglarda yorqin tasvirlaganligi bilan realistik adabiyot durdonasi hamdir. Zero, realistik ijod tabiati shundayki, san'atkor voqelikni oddiygina tasvirlab yoki u haqda mutlaq betaraf hikoya qilib qolmaydi. Realist san'atkor o'zi tasvirlayotgan voqelikni bilish, unga o'z munosabatini bildirishni ijodning birlamchi maqsadi deb biladi [4: B. 286]. Rus tanqidchisi V.G. Belinskiy bu haqda "Нам мало наслаждатъся - мы хотим знатъ: без знания для нас нет наслаждения" [1: B. 272] deb yozgan edi. Tanqidchining bu so'zlari ijodkor yozuvchiga ham kitobxon o'quvchiga ham birdek taalluqlidir. Yaxshi kitobxon romanda tasvirlanayotgan voqea-hodisalar shiddatinigina emas, balki $\mathrm{u}$ ifodalayotgan ma'noni, o'sha ma'no orqlali anglatmoqchi bo'lgan haqiqatni his etgandagina undan chinakam zavq ola biladi.

A. Cho'lponning "Kecha va Kunduz" asari ham kitobxonga ana shunday zavqni bera oladigan durdona romanlardan biridir. Roman o'zbek xalqining XX asr boshlaridagi hayotini badiiy gavdalantirgan bo'lib, unda adib o'sha davr muhiti: kishilarning savodsizligi, ayollarning huquqsiz va erksizligi bilan bir qatorda, o'zbek xalqiga xos fazilatlarni, ana'na va qadryatlarni, kishilar o'rtasidagi munosabatlarni ham tasvirlab beradi. Ushbu maqolada biz asar qahramonlaridan foyfalangan holda, XX asr boshlarida o'zbek xonadonlaridagi er-xotin munosabatlarini tahlil qilmoqchimiz.

\section{ASOSIY QISM}

Professor M. Qo'shjonov romanning boshidan oxirigacha hamma qismlarini va obrazlarini bir-biriga bog'lab turgan yagona obraz o'rni ancha zaif [5: B. 8] deb topgan bo'lsada , R. Otayev va 0 . Sharafiddinovlar qarashlarida romanning bosh qahramoni Zebi degan qarash ustivorroq mavqeyga ega [3: B. 79], [6: B. 64]. Bizningcha ham, Zebi roman bosh qahramoni sifatida o'zida o'zbek ayollariga xos xislatlarni jamlagan obrazdir. Zebi va Akbarali mingboshining er-xotinlik munosabatlariga nazar tashlasak buning yana bir bor guvohi bo'lamiz.

"- Tur, hay! - dedi Zebiga.

Zebi yotgan joyidan turib, fonarni balandlatdi.

- Dasturxonni yoz! Yegulik narsang bormi?

- Ha, kosada osh bor.

- Ber buyoqqa 
Zebi erining mast ekanligini bilsa-da, uning ovozidagi bu g'ayritabiiy qo'rslik va qattiqlikka hayron boldi, boshini ko'tarib, unga tikildi, "o'zimi, boshqami?” deganday...

- Nimaga menga qaraysan? Yo mastmisan, sintaloq? - dedi mingboshi.

Zebi indamasdan dasturxonni yoyib oshni qo'ydi'. [2: B. 294-295]

Dialog shaklidagi ushbu parchada muallif Zebi timsolida o'zbek ayollariga xos bo'lgan qator fazilatlarni ko'rsatib bergan. Ulardan birinchisi Zebining o'zbek ayollari singari er-xotin munosabatlarida o'z eriga itoatkorligi va turmush o'rtog'ining barcha gaplarini so'zsiz bajarishidir. Akbarali kelgandan so'ng Zebi darhol o'rnidan turadi va uning buyruqlarini bajara boshlaydi. Akbarali mingboshi to'ydan keyin ilk bor unga bunday qo'pol munosabatda bo'layotgan edi. Zebi bundan hayratlandi va boshini ko'tarib unga tikilib qoladi. Erkak kishi gapirganda, ayol boshini egib, ko'zlarini yerga olishi ayolning o'z eriga bo'lgan hurmatini bildirar edi. Erkak kishini yuziga boqish, ko'zlariga tik qarash unga nisbatan hurmatsizlik va betgachoparlik hisoblanardi. Zebi o'n besh yoshli juvon bo'lishiga qaramasdan, sha'riatning bu qonunini yaxshi bilardi. U hurmatsizlik yuzasidan emas balki hayratdan unga tikilib qolgan edi. Ammo, turmush o'rtog'i - Akbarali uni “-Nimaga menga qaraysan? Yo mastmisan, sintaloq?"deb nohaq koyidi. Shunda ham Zebi indamadi, e'tiroz bildirmadi, aksincha dasturxonni yozdi, o'z ishini davom ettirdi. Zebining o'z xojasiga gap qaytarmagani, e'tiroz bildirmagani uning o'zbek ayollariga xos iboli hayoli va andishali ekanini yana bir bor namoyon qiladi.

Endi asarning kulminatsion nuqtasi bo'lgan sud voqeasiga nazar tashlasak. Zebi o'sha zaharlanish hodisasidan beri karaxt bir holda bo'lardi. Uning miyasi falajga yo'liqqan kabi edi [7: B. 190]. U butun bir so'roq, tergov, konvoy, sud va zakunchilarga ajib bir loqaydlik bilan - xuddi jonsiz odamdek qarardi. U o'zini himoya qilishni, nima deb gap qaytarishni o'ylamasdi ham. U berilgan savollarga ro'y-rost javob berdi xolos. Uzoq savol-javobdan so'ng sud raisi: "- Demak, eringizni o'zingiz o'ldirdingiz?” [2: B.307] degan xulosaga keladi. Zebi shunda qattiqroq va cho'zibroq javob beradi.

“- Yo'o'q!.. Olibmanni o’z erimni o'ldirib? Voy, olaqolay, men shunaqa dermidim?" [2: B. 307] Bu javobda Zebining tabiiyligi, beg'uborligi shundoqqina ko'rinib turibdi. U Akbaralini shar'iy eri deb biladi. Uning faqatgina so'zi emas, balki har qanday xoxishi ham Zebi uchun qonun. Garchi, Zebi Akbaraliga o'zi xoxlab turmushga chiqmagan bo'lsada, uning har qanday istagini bajo keltirishga unning ko'nglini olishga har doim tayyor. Va buni o'zi xotin kishining ham farzi, ham qarzi deb hisoblaydi. O‘z erini o'ldirishni esa hayoliga ham keltirmaydi. Ammo sud raisi shar'iatning bu qonunini tushunmaydi, Zebi esa sud ishini. Ular ikki xil tilda gaplashadi. Zebi o'zi ulg'aygan erksiz sovuq muhitdan yanada sovuqroq joy Akbarali mingboshining xonadoniga keladi. Shunda ham isyon ko'tarmaydi - taqdirga tan beradi. Ammo, endi undanda sovuqroq joy Sibirga ketayotganini tasavvur ham qilolmaydi. Adib ikki xildagi dunyoqarashning bunday to'qnashuvini o'ta mohirona va tabiiy holda tasvirlab bergan. Biz Zebi timsolida hali balog'at yoshiga yetmagan qizni o'zini ayollar kabi hisoblab, o'z bo'yniga shunday ma'suliyatni olganini ko'ramiz. Zebining o'zini bunday tutishi, nafaqat uning o'zbek ayollariga xos sadoqatini, balki ma'suliyatni chuqur his etishini ham ko'rsatadi.

Er-xotin munosabatlarida o'zbekona sadoqatning yana bir ko'rinishini epizodik personajlardan biri Miryoqubning xotinida ham ko'rishimiz mumkin.

"- Nimaga yiglaysan? - dedi Miryoqub.

- Shahardan bugun keldingiz. Endi ikki oyga ketaman, deysiz... Yosh-yosh bolalaringiz bo'masa ham mayliydi...

- Bolalarimga yetarli pul qoldirdim. Baqqolga tayinlab qo'ydim, nima desang berib turadi. Ikki oy uzoqmi? Lahzada o'tadi-ketadi”' [2: B. 200]. 
Miryoqub va xotini o'rtasidagi munosabatlardan o’zbek ayolining eriga naqadar sadoqatli va itoatkor ekanligini ko'rishimiz mumkin. Xotinning oxirigacha ayta olmay qolgan gaplaridan erining xiyonatini, oilasini tashlab ketayotganini bilsada, uni erining o'ziga aytolmayotganini anglaymiz. U ko'rib bilib turgan haqiqatlarni ham ichidan chiqarmaydi. Erining xiyonatini farzandlari uchun ichga yutadi. Dardlarini faqat ko'z yoshlar orqali chiqaradi va yig'i bilan ovunadi. U eridan ketmaslikni o'tinadi, ammo Miryoqub rozi bo'lmagach uni ortiq qistamaydi.

"- Gugurt ber. Mehmonxonaning chirog'ini yoqay.

- Men sizga ana u sóriga joy qilib qo'ydim. Ketadigan kuningiz bolalaringiz bilan birga yoting endi.

- Sen o'zing bilasanki, men ichkarida yotolmayman”. [2: B. 200].

$\mathrm{Bu}$ qisqagina parcha ayolning naqadar mehrga zor ekanini, eridan najot kutayotganini ko'rsatadi. Miryoqub bu mehrga munosib javob qaytarmaydi. Shunda ham u mehrni tashqaridan izlamaydi, eriga jahl qilmaydi, aksincha uning xoxishlarini bajarishga harakat qiladi. Xotin erining barcha qilmishlarini bilsada, boshqa bazi ayollar (Poshshaxon, Sultonxon) singari xiyonat yoliga kirmaydi va xatto, buni o'ylamaydi ham. U oilasini deb yashaydi, farzandlari uchun jonini berishga ham tayyor. U haqiqiy o'zbek ayoli, o'zbek onasi timsolidir.

"- Qachon uyg'otay? Yo'lga nimalarni tugib qo'yay? - deb so'radi xotin xo'rlikdan ovozi tutilgan holda.

- Hech narsa kerak emas. O’zim uygonaman.

O'rta eshikjuda ohistalik bilan yopildi. So'ngra yana o'shanday ohistalik bilan zanjir solindi. Undan keyin, ayvonchaga yetib qolgan Miryoqubning quloqlariga yana o'sha tomondan ho'ngrab yiglagan bir ovoz eshitildi..."[2: B. 200-201].

Xotinning ichi olov bo'lib yonardi. Qalbidagi bu olovni o'chirishga kuch topolmasdi. Shunda ham u o'zini tutdi: safar uchun eriga nimalar kerakligini so'radi. Eridan sovuq javob eshitsa ham, u jahl qilmadi. Eshikni ham ohista, sekingina yopdi, zanjirni ham yana o'shanday ohistalik bilan soldi. Ammo, qalbiga so'zi o'tmadi, o'zini yig'idan tutolmadi. Bu holatini hech kimga, xattoki eriga ham ko'rsatolmadi. Dardini hech kimga doston qilmadi. Aybini tushunolmay, alamini yig'idan oldi. Hasratini ichiga yutdi. Biroq ko'z yoshlariga kuchi yetmadi...

Biz, Zebida yosh o'zbek ayolini, Miryoqubning xotinida o'rta yoshdagi o'zbek ayoli ko'rsak, yoshi katta o'zbek ayolini, shubhasiz, Qurbonbibi timsolida ko'rishimiz mumkin. Yosh o'zbek ayoli sodda, beg'ubor, samimiydir. U o'z eriga itoatkor, erining har bir so'zini, har qanday xoxishini bajarishni o'zining ham farzi, ham qarzi deb biladi. O'rta yoshda esa u farzand uchun yashaydi. Hayotning barcha qiyinchiliklariga farzandi, ota-onasi uchun bardosh beradi. O'z hayotini butunlay unutadi. Yoshi ulg'ayganda esa u hayotning ancha-muncha achchiq-chuchigini tortadi. Atrofdagilarning, ayniqsa turmush o'rtog'ining xarakteri, qarashlari va nozik tomonlarini ham juda yaxshi bilib, xattoki unga ta'sir o'tkazish yo'llarini ham topa oladi. Biz bunga quyidagi Razzoq so'fi va Qurbonbibi o'rtasidagi suhbatda ham yana bir bor amin bo'lishimiz mumkin.

"Sukut uzoqqa cho'zilgandan keyin Qurbonbibi endi bu safar jiddiy bir chehra bilan:

- Nimaga indamaysiz? Xo'p deng! Katta odam, uyat bo ladi. Bir yaxshi xotinlari, bir otincha qizlari borki... O'zlarini bo'Isa, o'zingiz bilasiz, - dedi.

So'fi negadir:

- Bilaman, Fitna, bilaman! - deb qo'yib, yana jim boIdi.

Endi Qurbonbibi yana ham jiddiylashdi:

- Bo'Imasa, "yo'q" deng. Saltanatxonga javob beray, ketsin! Azonda kelgan edi. Shundan so'ng so'fining tili aylandi:

- Shoshma, Fitna, "yo'q”, dema, mayli, bora qolsin..."[2: B. 21]. 
Bilamizki, Zebining otasi - Razzoq so'fi ishlamay tishlovchi, tekinxo'rlik va ta'magirlik ortidan kun ko'ruvchi, kulishi kasal odamning kulishiday og'ir, xotin-xalajni odam o'rnida ko'rmaydigan, ziqna odam. U boshliq uy qizi va xotini uchun zindon. O’zi esa shu zindonning ko'zi ko'r, qulog'i kar qorovulidir. Xattoki, muallif unga "Bu odam, jadidnamo bir hamshaharining deganidek, "ko'rgazmaga qo'yilaturgan antiqa mahluqlardan edi" [2: B.9] deb ta'rif beradi. Huquqlari toptalgan jamiyatda, shunday og'ir va qo'rs odam bilan bir uyda yashash Qurbonbibi uchun oson emas, albatta. Ammo, u taqdirga tan bergan, o'zining yashash prinsiplarini yaratgan. U qa'tiatli, zukko o'zbek ayoli. U xattoki, Razzoq so'fidek insonning nozik joylarini yaxshi biladi, unga ta'sir o'tkaza oladi. Xotin-xalaj oldida og'iz ochib til qaldiratishni ravo ko'rmaydigan so'fini, bazida tillarini burro qilib sayratadi. Biz bunga yuqoridagi parchada ham guvoh bo'lamiz.

Zebining dugonasi Saltanat Enaxonlarnikiga Zebini olib borish niyatida keladi. Onasi Qurbonbibi so'fining bunga aslo rozi bo'lmasligini biladi va Zebiga ruxsat olib berish maqsadida Xalfa eshon haqidagi yolg'onni o'ylab topadi. O'zi o'ylab topgan Xalfa eshonni uning oilasini xuddi bordek tabiiy qilib ta'riflaydi. Ammo, Razzoq so'fi uchun eshonning bir yaxshi xotini ham, otincha qizi ham qiziq emas. Hamma gap uning Xalfa eshon ekanligida. Qurbonbibi buni juda yaxshi biladi va shuning uchun qo'rqmasdan qa'tiat bilan gapiraveradi. Asarda Zebi ham bunga iqror bo'lib: "Eshon, desangiz, otam o Iganini ham bilmaydi... Xudo muni eshonlar uchun yaratgan." [2: B. 21] degan edi.

Biroq, Qurbonbibining onalik tuyg'usi, farzandiga bo'lgan muhabbati uning ko'zlarini ko'r qildi, uning mana shu kichik yolg'oni yakka-yu yagona, suyukli qizi Zebining achchiq qismatiga sabab bo'lib qolishini u hayoliga ham keltirmadi... Nima bo'lganda ham u farzandparvar o'zbek onasi timsolidir.

Zebini, Miryoqubning xotinini va Qurbonbibini barcha yoshda birlashtirib turgan, yoshi o'tgan sari ham ularni tark etmagan ularning sharqona fazilati bu - ularning o'z eriga itoatkorligi, erining hurmatini joyiga qo'yishi, erining ruxsatisiz biror ish qilmasligi va óz eriga andishali ekanidir.

\section{XULOSA}

Xulosa qilib shuni aytish mumkinki, maqolada A.Cho'lponning "Kecha va Kunduz" romanidagi qahramonlardan foydalanilgan holda er-xotin munosabatlari tahlil qilindi. Er-xotin munosabatlari tahlilida uch xil yoshdagi ayollar olinib, ulardagi o'zbek ayollariga xos jihatlar, yoshga ko'ra farqli jihatlar va umumiy bo'lgan jihatlar ko'rsatildi. Zebi yosh, Miryoqubning xotini o'rta yosh va Qurbonbibi katta yoshdagi o'zbek ayoli sifatida talqin etildi.

\section{FOYDALANILGAN ADABIYOTLAR RO'YXATI:}

1. Белинский В.Г. Полное собрание сочинений. - М.: 1955. - Т. 6. - С. 661-272.

2. Абдулхамид Чўлпон. Кеча ва кундуз: Роман - Т.: Янги аср авлоди, 2013 - Б. 332.

3. Отаев Р. Тонг юлдузи шуьлалари. - Шарқ юлдузи. - 1989. - Б. 208.

4. Куронов Д. Чўлпон насри поетикаси. Т.: “Шарқ”, 2004. - Б. 288.

5. Кўшжонов М. Кеча ва Кундуз романида образлар тизмаси // Ўзбек тили ва адабиёти. - 1992. - Б. 128.

6. Шараффиддинов О. Чўлпон. - Т.: Фан ва турмуш, 1991 - Б. 153.

7. Каримов Н., Мамажонов С., Назаров Б ва бошқалар. ХХ аср ўзбек адабиёти тарихи: Дарслик. - Т.: Ўқитувчи, 1999. - Б. 554. 